\title{
Influence de l'addition de différentes matières fertilisantes sur la biodisponibilité du cadmium, du manganèse, du nickel et du zinc contenus dans un sol sableux amendé par des boues de station d'épuration
}

Christian JUSTE \& Paul SOLDÂ

I.N.R.A., Station d'Agronomie, Centre de Recherches de Bordeaux, B.P. 13I, F 33140 Pont-de-la-Maye

En mettant en œuvre une culture expérimentale de Ray-grass d'Italie conduite en serre, en petits vases de végétation, on a mesuré l'influence de divers matériaux (engrais azoté ammoniacal, carbonate de calcium, tourbe blonde acide, hydroxyde de fer, grenailles d'acier) sur la biodisponibilité des métaux lourds ( $\mathrm{Cd}, \mathrm{Ni}, \mathrm{Mn}, \mathrm{Zn}$ ) d'un sol provenant d'un dispositif expérimental de plein champ oủ ont été épandues des boues à forte charge métallique.

La biodisponibilité des métaux, estimée par leur concentration dans le végétal, est augmentée par l'acidification résultant de l'addition de l'engrais azoté ammoniacal et diminuée par l'effet alcalinisant du carbonate de calcium. Ces variations de biodisponibilité sont bien corrélées avec les quantités de métal extraites du sol par $\mathrm{CaCl}_{2}$ ou $\mathrm{BaCl}_{2}$ en solution décinormale mais pas avec celles extraites par $\mathrm{HCl} 0,1 \mathrm{~N}$, en raison de la trop grande agressivité de ce réactif.

Les réactifs chimiques mis en œuvre ne permettent cependant pas d'interpréter l'action dépressive exercée sur la biodisponibilité du cadmium par la tourbe et surtout par les différentes sources de fer experimentées.

Lors des premières coupes, l'action bénéfique de l'azote de l'engrais ammoniacal ne permet pas de mettre en évidence un effet des métaux sur la production de matière sèche du ray-grass ; cette dernière est diminuée par la carence en manganèse induite par l'apport de $\mathrm{CaCO}_{3}$. Par contre, pour la dernière coupe, il existe une bonne relation entre la décroissance des concentrations en $\mathrm{Cd}$ et $\mathrm{Ni}$ du végétal, provoquée par l'ajout de tourbe, d'hydroxyde de fer ou de grenaille d'acier, et la plus grande production de matière sèche enregistréc en présence de ces 3 amendements.

L'effet dépressif important exercé par les différentes sources de fer sur la biodisponibilité de métaux toxiques comme le cadmium pourrait avoir des retombées pratiques intéressantes pour la lutte contre la pollution métalique des sols cultivés.

Mots clés additionnels: Métal lourd, tourbe, acidification, chaulage, hydroxyde de fer. soil as a result of ammonium sulphate, acid peat, lime or iron compound addition.

The effect of various materials (ammonium sulphate, iron hydrous oxide, calcium carbonate, acid peat and steel shot) on the bioavailability of heavy metals (Cd, Ni, $\mathrm{Zn}, \mathrm{Mn}$ ) pre-existing in a sludge treated soil was investigated using a pot experiment procedure with Italian ryegrass as test-plant. Metal bioavailability (expressed as the plant metal content) was increased as a result of acidification due to ammonium sulphate addition (tables $2,4,5$ and 9) ; calcium carbonate incorporation increased the soil $\mathrm{pH}$ and consequently diminished heavy metal content in the plant. The changes in bioavailability were strongly correlated with amounts of metals solubilized using $0.1 \mathrm{~N} \mathrm{BaCl}_{2}$ or $0.1 \mathrm{~N} \mathrm{CaCl}_{2}$ as extracting solutions (tables 6,7 and 9). However it was not possible, using chemical extractants, to explain the drop of $\mathrm{Cd}$ bioavailability due to acid peat, iron hydrous oxide or steel shot addition to soil. For the first cut of the ryegrass, the fertilizing power of ammonium nitrogen probably alleviated a possible detrimental effect originating from the increase in metal bioavailability and, thus, enhanced the yield of ryegrass (table 3). In contrast, $\mathrm{Mn}$ deficiency induced by $\mathrm{CaCO}_{3}$ incorporation into the soil prevented an expected beneficial effect due to a lack of toxic metal bioavailability and, on the contrary, decreased dry matter production. However, it was obvious that peat acid, iron hydrous oxide or steel shot addition to soil resulted in a noticeable increase of dry matter yield at the last cuts of the ryegrass; this bencficial effect was strongly correlated with a lack of cadmium and nickel content in ryegrass (table 10). The drop of bioavailability of such potentially 
hazardous metals as cadmium in soil treated with various iron components represents an interesting finding with a view to a better control of soil metal contamination.

Additional key words : Heavy metals, peat, acidification, liming, iron hydrous oxide.

\section{INTRODUCTION}

La biodisponibilité d'un élément, défini dans le cas présent comme son aptitude à passer d'un compartiment quelconque du sol dans un végétal, est principalement sous la dépendance de l'état et de la localisation de cet élément. Les facteurs susceptibles d'agir sur ces deux caractéristiques peuvent donc modifier la biodisponibilité de tout élément du sol transférable dans le règne végétal.

Il est couramment admis que le $\mathrm{pH}$ du sol constitue le principal facteur qui détermine la biodisponibilité des métaux.

Certaines pratiques agricoles comme l'apport de fumures azotées ammoniacales (AMES, 1921) peuvent conduire à une acidification du sol et par là-même provoquer l'apparition de manifestations de toxicité. Un grand nombre de travaux ont été conduits pour évaluer l'importance de ce phénomène en fonction de la nature et des doses d'engrais azoté appliquées et ses conséquences sur la mobilité de certains métaux préexistant dans le sol tels que Al, Mn, Fe (ABRUNA er al., 1964; AYRES et al., 1965; FISKELL, 1965; ADAMS et al., 1967; ADAMS \& PEARSON, 1969 ; SluiJMANS, 1970 ; Siman et al., 1971 ; HoYt \& HenNing, 1982). Les travaux de même nature, relatifs à d'autres métaux apportés par certaines matières fertilisantes, sont à notre connaissance plus rares (FU-HSIAN CHANG \& BROADBENT, 1980 ; INTRAWECH et al., 1982 ; JUSTE \& TAUZIN, 1986) sans doute en raison de l'intérêt plus récent qui leur est accordé.

$\mathrm{Si}$ une variation de $\mathrm{pH}$ peut modifier la biodisponibilité des métaux en agissant sur leur spéciation et leur localisation, il peut être également envisagé d'intervenir sur cette caractéristique en ajoutant au sol des phases organiques ou minérales qui fonctionnent comme adsorbants (tourbes, silicates, hydroxydes...).

Le but du travail présenté dans cette note est d'évaluer, grâce à une expérience en vases de végétation conduite en serre, les variations de biodisponibilité d'un certain nombre de métaux dont l'accumulation dans le sol résulte de plusieurs années d'application en plein champ de boues urbaines très polluées par des métaux. Ces variations de biodisponibilité ont été intentionnellement provoquées en ajoutant au sol soit du sulfate d'ammonium ou du carbonate de calcium agissant comme modificateurs du $\mathrm{pH}$, soit de la tourbe brute ou divers hydroxydes de fer agissant comme phase de rétention des métaux présents dans le sol. Elles ont été appréciées par la mesure de la concentration en métaux dans les parties aériennes d'un ray-grass d'Italie utilisé comme plante-test. Il est clair que ce test biologique n'a pas une valeur prédictive généralisable ; il permet cependant, pour les conditions bien précises de l'expérience décrite, de comparer l'effet des différents traitements sur cette caractéristique qui intègre le comportement $\mathrm{du}$ métal dans le sol et celui de la plante. Cette évaluation de la biodisponibilité effective des métaux a été rapprochée des quantités extraites par quelques réactifs chimiques: $\mathrm{CaCl}_{2}$ (SAUERBECK \& STYPEREK, 1985), $\mathrm{BaCl}_{2}$ (MeguelatTr et al., 1983) et $\mathrm{HCl}$ dilué (LiNÈres \& JUSTE, 1980), employés pour estimer la fraction du métal du sol à laquelle la plante serait susceptible d'accéder le plus facilement.

\section{MATÉRIEL ET MÉTHODES}

\section{A. Sol}

Le sol a été prélevé en avril 1985 dans les parcelles d'un dispositif mis en place en 1976 au Domaine expérimental de l'I.N.R.A. à Couhins (Gironde). Ce dispositif, décrit en détail par ailleurs (JUSTE \& SOLDÂ, 1985), comporte les 4 traitements suivants:

- Témoin - Fertilisation minérale seule ;

- Apport de $10 \mathrm{t} /$ ha de matière sèche de boues tous les ans;

- Apport de $100 \mathrm{t} /$ ha de matière sèche de boues tous les 2 ans;

- Apport de $10 \mathrm{t} / \mathrm{ha}$ de matière sèche de fumier tous les ans.

Les apports de produits organiques ont été interrompus en 1980 mais le dispositif a été maintenu afin de suivre les arrière-effets des divers traitements sur le comportement du végétal et les caractéristiques du sol.

Les 5 parcelles de chaque traitement $\left(18 \mathrm{~m}^{2}\right)$ portent depuis le début de l'expérience une monoculture de maïs (variété INRA 260). La fertilisation N-P-K est ajustée à un même niveau dans les traitements: témoin, $10 \mathrm{t} / \mathrm{ha} / \mathrm{an}$ de boues et $10 \mathrm{t} / \mathrm{ha} / \mathrm{an}$ de fumier, le traitement $100 \mathrm{t} / \mathrm{ha} / 2$ ans recevant les mêmes compléments minéraux que ceux éventuellement ajoutés dans les parcelles $10 \mathrm{t} / \mathrm{ha} /$ an de boues.

Le prélèvement de sol destiné à l'expérience a été réalisé dans les 5 parcelles qui ont reçu au total $50 \mathrm{t} / \mathrm{ha}$ de matière sèche de boues en 5 ans. Les principales caractéristiques du sol et de la boue sont indiquées dans le tableau 1.

Les échantillons destinés à l'expérience ont été tamisés à $4 \mathrm{~mm}$.

\section{B. Matériaux ajoutés au sol}

En vue de provoquer des variations de la biodisponibilité des métaux, on a ajouté les produits suivants (préalablement pulvérisés) en les mélangeant intimement au sol : 
TABLEAU 1

Composition du sol et de la boue expérimentés (par rapport à la matière sèche).

Mean soil and senage sludge characteristics.

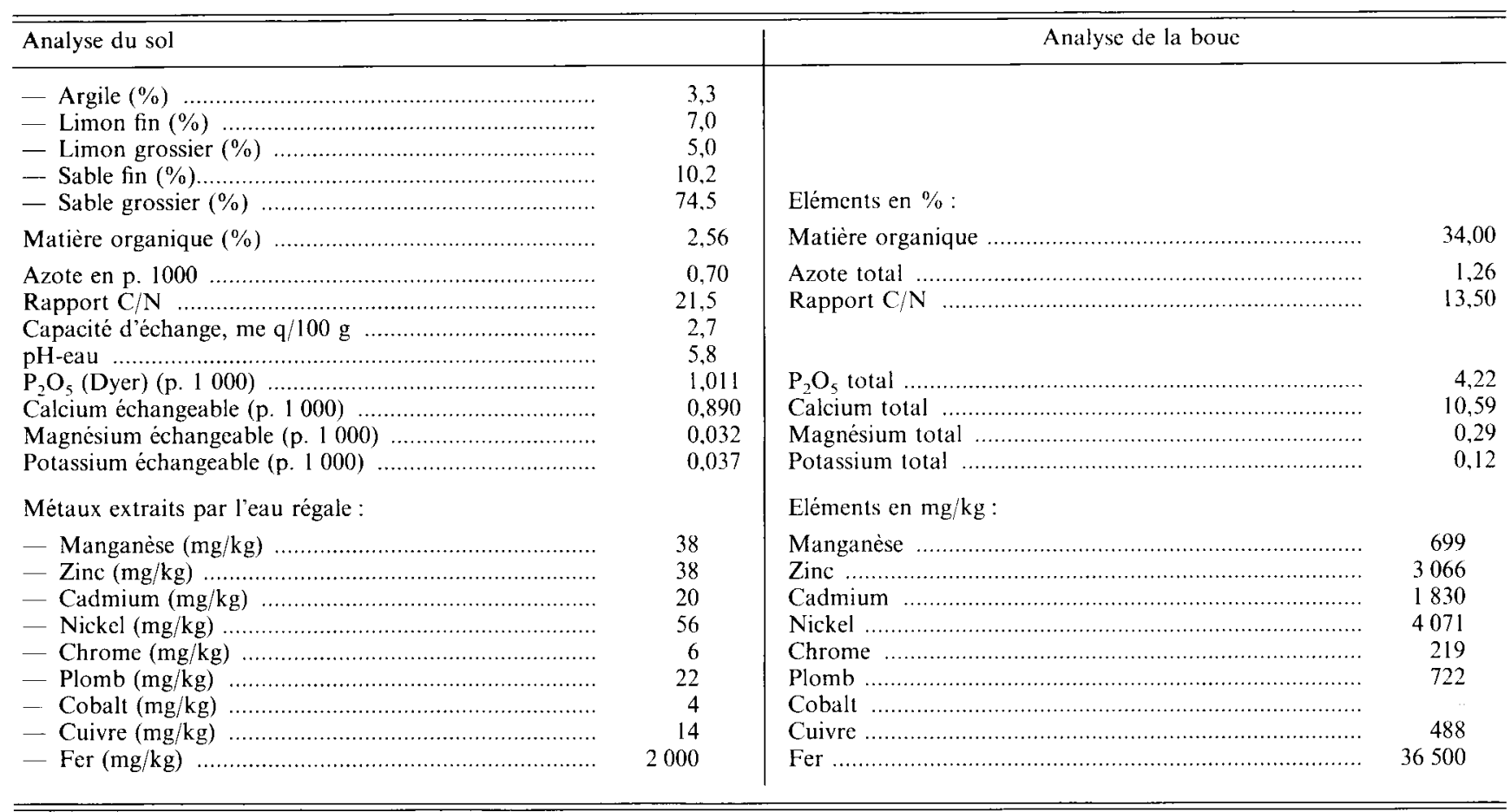

- sulfate d'ammonium (équivalent de $500 \mathrm{~kg} / \mathrm{ha} \mathrm{N}$ );

- carbonate de calcium (équivalent de

$4000 \mathrm{~kg} / \mathrm{ha} \mathrm{CaO}$ ) ;

- tourbe brute acide Floratorf ( 1 p. 100 en poids) ;

- hydroxyde de fer ( 1 p. 100 en poids);

- carbonate de calcium et tourbe brute acide (équivalent de $4000 \mathrm{~kg} / \mathrm{ha} \mathrm{CaO}$ et 1 p. 100 en poids);

- grenailles d'acier ajoutées à raison de 1 p. 100 en poids.

L'hydroxyde de fer a été préparé au laboratoire en précipitant par de la soude une solution de sulfate ferreux. Le précipité obtenu a été lavé plusieurs fois à l'eau bipermutée, séché à $60^{\circ} \mathrm{C}$ puis broyé finement.

Les grenailles d'acier employées, de type angulaire, sont couramment utilisées comme matériau abrasif; leur granulométrie moyenne est inférieure à $450 \mu$ et elles renferment comme principales impuretés: du carbone $(0,9$ p. 100$)$, du silicium $(0,9$ p. 100$)$, du soufre $(0,05$ p. 100$)$, du phosphore $(0,05$ p. 100$)$ et du manganèse $(0,7 \mathrm{p} .100)$.

\section{Culture expérimentale}

Elle a été conduite en petits pots ( 5 répétitions) contenant chacun $1 \mathrm{~kg}$ de terre fine, placés en serre munie d'un dispositif de refroidissement par ventilation forcée d’air humidifié. La culture-test est constituée par du Ray-grass variété Sabrina, semé à raison de $1 \mathrm{~g}$ par pot.

Avant semis, on a apporté une fertilisation standard de fond comportant l'équivalent de $150 \mathrm{~kg} / \mathrm{ha} \mathrm{K}_{2} \mathrm{O}$ (chlorure de potassium) et de 150 unités/ha d'azote (ammonitrate 33 p. 100); par ailleurs, il a été jugé inutile d'effectuer une incorporation d'anhydride phosphorique en raison de l'enrichissement notable en cet élément résultant de l'apport des boues. Après mélange des sols avec les différentes matières fertilisantes et avant semis, les pots ont subi une phase de maturation en serre durant 5 semaines, au cours desquelles ils ont été régulièrement arrosés à 75 p. 100 de la capacité de rétention et scarifiés.

D'avril à octobre, 6 coupes successives de ray-grass ont été effectuées à raison d'une coupe par mois environ. Après chaque coupe à l'exception de la seconde, $25 \mathrm{ml}$ d'une solution nutritive ne comportant pas d'oligo-éléments ni de phosphore ont été ajoutés à chaque pot $(\mathrm{N}=350 ; \mathrm{K}=110 ; \mathrm{S}=60 \mathrm{meq} / \mathrm{l})$.

\section{Déterminations réalisées}

On a déterminé à chaque coupe le poids de matière sèche de ray-grass produit.

Seules les coupes $n^{\circ} 1,2$ et 6 ont été analysées; après minéralisation par voie sèche et reprise des cendres par $\mathrm{HNO}_{3}$ à 5 p. 100, on a dosé les éléments suivants:

- $\mathrm{Cu}, \mathrm{Fe}, \mathrm{Mn}, \mathrm{Zn}, \mathrm{Cd}, \mathrm{Ni}$ par spectrophotométrie d'absorption atomique en flamme ;

- $\mathrm{Cr}$ et $\mathrm{Pb}$ par spectrophotométrie d'absorption atomique au four graphite :

- Les pH ont été mesurés dans une suspension de sol (20 g/50 $\mathrm{ml}$ d'eau) après 4 mois d'incubation en serre dans les conditions de culture du ray-grass.

Dans ces mêmes échantillons, on a procédé également à la détermination de la fraction des métaux $(\mathrm{Mn}, \mathrm{Zn}$, $\mathrm{Cd}, \mathrm{Ni}$ ) extractible par $\mathrm{BaCl}_{2} 0,1 \mathrm{~N}, \mathrm{CaCl}_{2} 0,1 \mathrm{~N}$ et $\mathrm{HCl} 0,1 \mathrm{~N}$ par agitation de $10 \mathrm{~g}$ de terre durant $2 \mathrm{~h}$ au contact de $50 \mathrm{ml}$ de chacun de ces réactifs. Après filtration sur papier, tous les métaux ont été dosés par spectrophotométrie d'absorption atomique en flamme. 


\section{RÉSULTATS OBTENUS}

\section{A. pH du sol (tableau 2)}

Compte tenu de la texture sableuse du sol, l'acidification résultant de l'ajout de $500 \mathrm{~kg}$ d'azote sous forme ammoniacale est notable. On observe également une action acidifiante significative de l'hydroxyde de fer. Par contre, l'ajout de carbonate de calcium et, dans une beaucoup moindre mesure, celui de grenailles d'acier ont entrainé une élévation du $\mathrm{pH}$. On peut observer également que l'addition conjointe de tourbe et de carbonate de calcium n'a que peu modifié l'effet alcalinisant exercé par ce dernier produit.

TABLEAU 2

Effot des differents amendements sur le $\mathrm{pH}$ du sol. Effect of fertilizer addition on soil $\mathrm{pH}$.

\begin{tabular}{ll}
\hline \hline Traitement & $\overline{\mathrm{H}}$ \\
\hline Témoin & $5.5 \mathrm{~d}$ \\
$500 \mathrm{~N}$ & $4.9 \mathrm{a}$ \\
$4000 \mathrm{CaO}$ & $7.4 \mathrm{~g}$ \\
$4000 \mathrm{CaO}+$ tourbe & $7.2 \mathrm{f}$ \\
Tourbe & $5.4 \mathrm{~cd}$ \\
Fe hydroxyde & $5.2 \mathrm{~b}$ \\
Fe grenaille acier & $6.4 \mathrm{~g}$ \\
\end{tabular}

Les valeurs qui ne sont pas suivies par une mème lettre sont significativement différentes au seuil de $5 \%$.

Within a column, means followed by the same letter atre no significantly different at the 0.05 level.

\section{B. Production de matière sèche du ray-grass}

Le tableau 3 montre que la production cumulée du ray-grass est accrue d'environ 20 à 25 p. 100 pour les 3 traitements comportant l'addition: soit de tourbe seule, soit d'hydroxyde de fer, soit de grenaille d'acier. On observe par contre une tendance à la manifestation d'un effet dépressif dù à l'ajout du carbonate de calcium.

L'analyse détaillée des résultats concernant les coupes successives (tabl. 3) ou de la cinétique de la production cumulée de matière sèche (fig. 1) met en évidence un effet dépressif significatif du carbonate de calcium lors des 2 premières coupes et un effet bénéfique, surtout net à la $I^{\text {re }}$ coupe, de l'apport de 500 unités d'azote sous forme ammoniacale. On constate enfin que, dès la $2^{\mathrm{c}}$ coupe, les 3 traitements comportant l'addition de tourbe seule, d'hydroxyde de fer ou de grenailles d'acier conduisent à une production supérieure de matière sèche, ce phénomène s'accentuant au cours du temps.

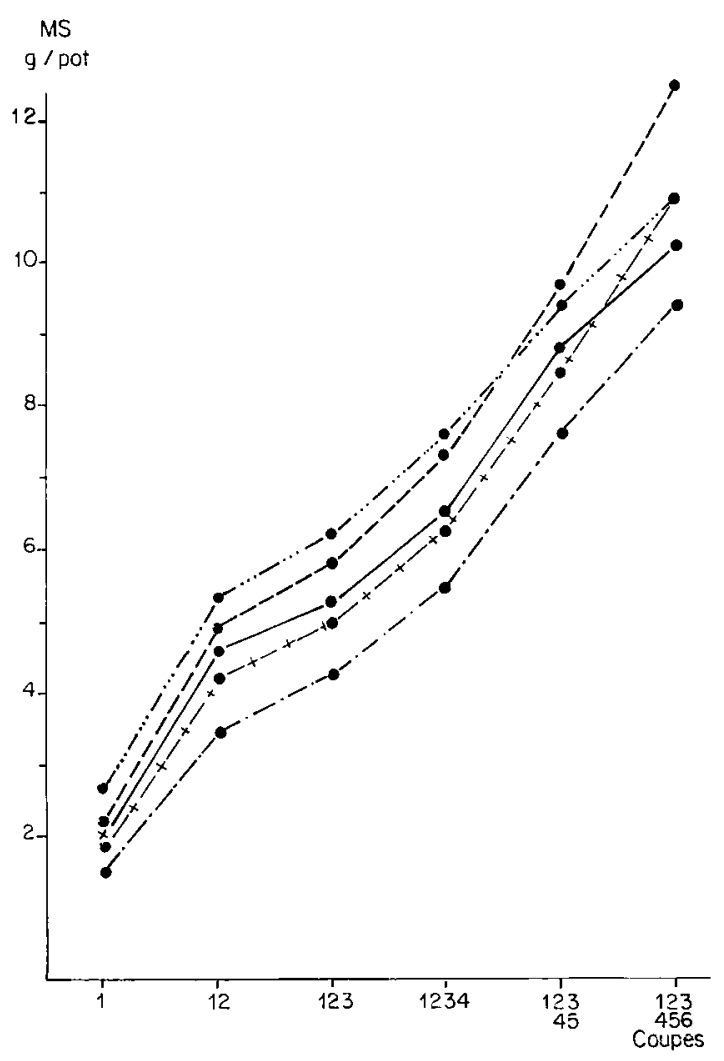

Figure 1

Prodaction (ammlé de ray-grass (g/pot de matiere seche).

Cumulated bield of regrass (g/pol of dry mater).

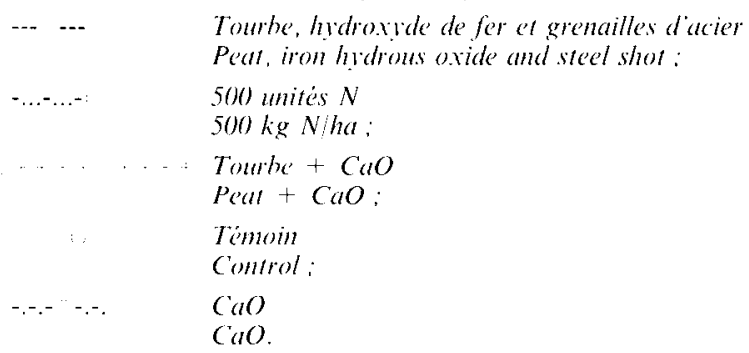

TABLEAU 3

Influence des différents amendements sur la production de matione sèche du Ray-grass (g/pot). Effect of various fertilizer additions on the dry matter vield of regrass $(\mathrm{g} / \mathrm{pol})$.

\begin{tabular}{|c|c|c|c|c|c|c|c|}
\hline Traitement & Coupe 1 & Coupe 2 & Coupe 3 & Coupe 4 & Coupe 5 & Coupe 6 & Prod. tot. \\
\hline Témoin & $2,08 \mathrm{bc}$ & $2.57 \mathrm{bc}$ & $0,70 \quad$ a & 1,25 a & $2,04 a b$ & $1,66 a$ & $10,30 \mathrm{ab}$ \\
\hline $500 \mathrm{~N}$ & $2,67 \mathrm{~d}$ & $2,71 \mathrm{~cd}$ & $0,88 \mathrm{abc}$ & $1,35 \mathrm{ab}$ & $1,86 \mathrm{a}$ & $1.48 \mathrm{a}$ & $10,96 \mathrm{~b}$ \\
\hline $4000 \mathrm{CaO}+$ tourbe & $1,91 \mathrm{~b}$ & $2,37 \mathrm{~b}$ & $0,73 \mathrm{ab}$ & $1,32 \mathrm{ab}$ & $2,25 \mathrm{bc}$ & $2.41 \mathrm{~b}$ & $10.98 \mathrm{~b}$ \\
\hline Tourbe & $2,19 \mathrm{c}$ & $2,86 \mathrm{~d}$ & $0,93 \mathrm{bc}$ & $1,62 \mathrm{c}$ & $2,25 b c$ & $2,40 \mathrm{~b}$ & $12,25 \mathrm{c}$ \\
\hline Fe Hydroxyde & $2,08 b c$ & $2,87 \mathrm{~d}$ & $0,98 \quad \mathrm{c}$ & $1,50 b c$ & $2,33 \mathrm{c}$ & $2,57 b$ & $12,33 \mathrm{c}$ \\
\hline Fe grenail. acier & $2,22 \mathrm{c}$ & $2,73 \mathrm{~cd}$ & $0.89 \mathrm{abc}$ & $1,53 \mathrm{bc}$ & $2,40 \mathrm{c}$ & $2,79 \mathrm{~b}$ & $12,55 \mathrm{c}$ \\
\hline
\end{tabular}

Dans une même colonne, les valeurs qui ne sont pas suivies par une même lettre sont significativement diflérentes au seuil de $5 \%$.

Within a column, means followed by the same letter are not significantly different at the 0.05 level. 


\section{Biodisponibilité des métaux}

Elle est exprimée par la concentration des divers métaux mesurée dans les parties aériennes du ray-grass. Seuls le manganèse, le zinc, le cadmium et le nickel donnent lieu aux variations les plus significatives en fonction du traitement et du numéro des coupes; on a donc analysé uniquement les résultats concernant ces éléments. Par ailleurs, les concentrations mesurées dans les coupes 1 et 2 étant très voisines, les données reportées dans les tableaux 4 et 5 ont été limitées aux coupes 1 et 6 .

\section{TABLEAU 4}

Effet des différents amendements sur la teneur en métaux de la première coupe de ray-grass

( $\mathrm{mg} / \mathrm{kg}$ matière sèche).

Effect of fertilizer addition on the metal concentration in the first cut of ryegrass

( $m g / k g$ dry matter basis)

\begin{tabular}{lcrcc}
\hline Traitement & $\mathrm{Mn}$ & $\mathrm{Zn}$ & $\mathrm{Cd}$ & $\mathrm{Ni}$ \\
\hline Témoin & $33 \mathrm{ab}$ & $130 \mathrm{c}$ & $18 \mathrm{c}$ & $61 \mathrm{~cd}$ \\
$500 \mathrm{~N}$ & $352 \mathrm{~d}$ & $204 \mathrm{~d}$ & $27 \mathrm{~d}$ & $99 \mathrm{f}$ \\
$4000 \mathrm{CaO}$ & $11 \mathrm{a}$ & $97 \mathrm{~b}$ & $12 \mathrm{~b}$ & $39 \mathrm{~b}$ \\
$4000 \mathrm{CaO}+$ tourbe & $11 \mathrm{a}$ & $91 \mathrm{~b}$ & $8 \mathrm{ab}$ & $32 \mathrm{ab}$ \\
Tourbe & $183 \mathrm{c}$ & $137 \mathrm{c}$ & $19 \mathrm{c}$ & $65 \mathrm{~d}$ \\
Fe Hydroxyde & $51 \mathrm{~b}$ & $117 \mathrm{c}$ & $16 \mathrm{c}$ & $55 \mathrm{c}$ \\
Fe grenail. acier & $22 \mathrm{ab}$ & $65 \mathrm{a}$ & $6 \mathrm{a}$ & $26 \mathrm{a}$ \\
& & & & \\
\hline
\end{tabular}

Dans une même colonne, les valeurs qui ne sont pas suivies par une même lettre sont significativement différentes au seuil de $5 \%$.

Within a column, means followed by the same letter are not significantly different at the 0.05 level.

\section{TABLEAU 5}

Effet des différents amendements sur la teneur en métaux de la sixième coupe de ray-grass

( $\mathrm{mg} / \mathrm{kg}$ matière sèche).

Effect of fertilizer addition on the metal concentration in the sixth cut of ryegrass

( $\mathrm{mg} / \mathrm{kg}$ dry matter basis).

\begin{tabular}{lrllc}
\hline \hline Traitement & $\mathrm{Mn}$ & $\mathrm{Zn}$ & $\mathrm{Cd}$ & $\mathrm{Ni}$ \\
\hline Témoin & $56 \mathrm{~b}$ & $242 \mathrm{ab}$ & $26 \mathrm{c}$ & $105 \mathrm{~d}$ \\
$500 \mathrm{~N}$ & $90 \mathrm{~d}$ & $351 \mathrm{~b}$ & $39 \mathrm{~d}$ & $127 \mathrm{f}$ \\
$4000 \mathrm{CaO}$ & $6 \mathrm{a}$ & $175 \mathrm{a}$ & $14 \mathrm{ab}$ & $57 \mathrm{ab}$ \\
$4000 \mathrm{CaO}+$ tourbe & $12 \mathrm{a}$ & $224 \mathrm{a}$ & $11 \mathrm{a}$ & $46 \mathrm{a}$ \\
Tourbe & $47 \mathrm{~b}$ & $210 \mathrm{a}$ & $20 \mathrm{~b}$ & $83 \mathrm{c}$ \\
Fe Hydroxyde & $46 \mathrm{~b}$ & $225 \mathrm{a}$ & $18 \mathrm{~b}$ & $97 \mathrm{~d}$ \\
Fe grenail. acier & $52 \mathrm{~b}$ & $138 \mathrm{a}$ & $15 \mathrm{ab}$ & $63 \mathrm{~b}$ \\
& & & & \\
\hline
\end{tabular}

Dans une même colonne, les valeurs qui ne sont pas suivies par une même lettre sont significativement différentes au seuil de $5 \%$.

Within a column, means followed by the same letter are not significantly different at the 0.05 level.

Les teneurs enregistrées dans le ray-grass à la $6^{\mathrm{c}}$ coupe sont, dans l'ensemble, beaucoup plus importantes que celles caractérisant la première. Ce phénomène peut être dû autant à l'accroissement au cours du temps de la colonisation de la masse de terre des vases de végétation par le système racinaire qu'à une amélioration de l'aptitude de la plante à mobiliser les métaux au cours de son vieillissement.

Outre le phénomène de concentration très important observé à la $6^{\mathrm{e}}$ coupe pour la quasi-totalité des métaux et des traitements, les résultats font apparaître, par rapport au témoin :

- une augmentation très significative de la concentration de tous les métaux dans le ray-grass cultivé sur le sol traité par l'engrais azoté ammoniacal ;

- une décroissance générale des concentrations en métaux du ray-grass issu des vases de végétation contenant le sol traité par le carbonate de calcium ou par l'addition conjointe de carbonate et de tourbe. Cette baisse est particulièrement intense dans le cas du manganèse.

D'une manière générale, hormis pour $\mathrm{Mn}$, l'addition de tourbe seule, d'hydroxyde de fer ou de grenailles d'acier provoque, par rapport au témoin, une baisse des concentrations en métaux du ray-grass. En présence de ces 3 phases adsorbantes, ce phénomène est particulièrement net dans le cas du $\mathrm{Cd}$ dont la concentration dans le végétal est abaissée à la $6^{\mathrm{C}}$ coupe, d'une manière aussi importante qu'à la suite d'un apport de carbonate de calcium.

\section{Extraction des métaux par $\mathrm{BaCl}_{\mathbf{2}} \mathbf{0 , 1} \mathrm{N}, \mathrm{CaCl}_{\mathbf{2}} \mathbf{0 , 1} \mathrm{N}$, HCl 0,1 N}

$\mathrm{BaCl}_{2} 0,1 \mathrm{~N}$ et $\mathrm{CaCl}_{2} 0,1 \mathrm{~N}$ (tabl. 6 et 7 ) extraient des quantités quasiment identiques de métaux; on constate cependant que $\mathrm{CaCl}_{2}$ possède un pouvoir d'extraction plus élevé dans le cas des sols traités par le carbonate de calcium.

TABLEAU 6

Effet des différents amendenents sur les quantités de métal extraites du sol par $\mathrm{BaCl}, 0,1 \mathrm{~N}$

( $m g / k g$ de sol)

Effect of various fertilizers on metal extraction by $0.1 \mathrm{~N} \mathrm{BaCl}$, ( $\mathrm{mg} / \mathrm{kg}$ of soil)

\begin{tabular}{lrrrr}
\hline \hline Traitement & $\mathrm{Mn}$ & $\mathrm{Zn}$ & $\mathrm{Cd}$ & $\mathrm{Ni}$ \\
\hline Témoin & $3,9 \mathrm{~b}$ & $7,2 \mathrm{c}$ & $9,4 \mathrm{~b}$ & $10,3 \mathrm{c}$ \\
$500 \mathrm{~N}$ & $11,2 \mathrm{f}$ & $14,4 \mathrm{f}$ & $12,2 \mathrm{c}$ & $15,5 \mathrm{~d}$ \\
$4000 \mathrm{CaO}$ & $1,1 \mathrm{a}$ & $0,6 \mathrm{a}$ & $4,3 \mathrm{a}$ & $2,0 \mathrm{a}$ \\
$4000 \mathrm{CaO}+$ tourbe & $1,0 \mathrm{a}$ & $0,7 \mathrm{a}$ & $4,3 \mathrm{a}$ & $1,8 \mathrm{a}$ \\
Tourbe & $6,1 \mathrm{c}$ & $10,7 \mathrm{~d}$ & $11,1 \mathrm{bc}$ & $12,8 \mathrm{c}$ \\
Fe Hydroxyde & $6,8 \mathrm{cf}$ & $4,7 \mathrm{~b}$ & $10,2 \mathrm{~b}$ & $11,3 \mathrm{c}$ \\
Fe grenail. acier & $9,9 \mathrm{df}$ & $1,2 \mathrm{a}$ & $4,9 \mathrm{a}$ & $5,1 \mathrm{~b}$ \\
& & & & \\
\hline
\end{tabular}

Dans une même colonne, les valeurs qui ne sont pas suivies par une même lettre sont significativement différentes au seuil de $5 \%$.

Within a column, means followed by the same letter are not significantly different at the 0.05 level.

Par comparaison avec le sol qui n'a reçu aucun amendement ou engrais, on observe un accroissement très significatif des quantités de métal extraites par les 2 réactifs pour les sols traités par $\left(\mathrm{NH}_{4}\right)_{2} \mathrm{SO}_{4}$ et, à l'inverse, un abaissement non moins significatif de la fraction extraite des sols additionnés de carbonate de calcium. L'incorporation de grenailles d'acier provoque une diminution très sensible des quantités extraites par les 2 solutions salines, à l'exception du manganèse dont la solubilité est tout au contraire très fortement augmentée par cet amendement minéral. L'ajout au sol de tourbe ou d'hydroxyde de fer n'a eu pratiquement 
TABLEAU 7

Effet des différents amendements sur les quantités de métal extraite's du sol par $\mathrm{CaCl}_{2} 0,1 \mathrm{~N}$

( $m g / k g$ de sol).

Effect of various fertilizers on metal extraction by $0.1 \mathrm{~N} \mathrm{CaCl}$ ? (mg/kg of soil).

\begin{tabular}{lrrrr}
\hline \hline Traitement & $\mathrm{Mn}$ & $\mathrm{Zn}$ & $\mathrm{Cd}$ & $\mathrm{Ni}$ \\
\hline Témoin & $4,5 \mathrm{~b}$ & $7,9 \mathrm{c}$ & $10,3 \mathrm{~b}$ & $11,8 \mathrm{c}$ \\
$500 \mathrm{~N}$ & $11,1 \mathrm{~d}$ & $13,9 \mathrm{f}$ & $12,0 \mathrm{c}$ & $15,9 \mathrm{f}$ \\
$4000 \mathrm{CaO}$ & $1,9 \mathrm{a}$ & $1,7 \mathrm{a}$ & $7,3 \mathrm{a}$ & $4,7 \mathrm{a}$ \\
$4000 \mathrm{CaO}+$ tourbe & $1,8 \mathrm{a}$ & $1,9 \mathrm{a}$ & $7,1 \mathrm{a}$ & $4,1 \mathrm{a}$ \\
Tourbe & $6,4 \mathrm{c}$ & $10,8 \mathrm{~d}$ & $11,7 \mathrm{~b}$ & $14,2 \mathrm{~d}$ \\
Fe Hydroxyde & $7,2 \mathrm{c}$ & $4,6 \mathrm{~b}$ & $11,1 \mathrm{~b}$ & $12,3 \mathrm{c}$ \\
Fe grenail. acier & $12,8 \mathrm{f}$ & $1,2 \mathrm{a}$ & $6,3 \mathrm{a}$ & $6,3 \mathrm{~b}$ \\
& & & & \\
\hline \hline
\end{tabular}

Dans une même colonne, les valeurs qui ne sont pas suivies par une même lettre sont significativement différentes au seuil de $5 \%$.

Within a column, means followed by the same letter are not significantly different at the 0.05 level.

aucune incidence sur l'extractibilité de $\mathrm{Cd}$ et $\mathrm{Ni}$ mais a accru celle de $\mathrm{Mn}$; le zinc a un comportement très particulier puisque sa solubilité dans $\mathrm{BaCl}_{2}$ ou $\mathrm{CaCl}_{2}$ augmente en présence de tourbe mais diminue considérablement en présence d'hydroxyde de fer.

Contrairement aux 2 solutions salines expérimentées, la solution d'acide diluée apparaît beaucoup moins discriminante vis-à-vis des différents traitements (tabl. 8) : le seul fait saillant est l'extraction par l'acide d'une quantité relativement importante de manganèse du sol additionné de grenailles d'acier, ce qui doit être mis en relation avec la richesse en $\mathrm{Mn}(0,7 \mathrm{p} .100)$ de l'amendement minéral.

TABLEAU 8

Effet des différents amendements sur les quantités de métal extraites du sol par $H(70,1 \mathrm{~N}$

( $m g / k g$ de sol).

Effect of various fertilizers on metal extraction by $0.1 \mathrm{~N} \mathrm{HCl}$ ( $m g / k g$ of soil ).

\begin{tabular}{lcccc}
\hline \hline Traitement & $\mathrm{Mn}$ & $\mathrm{Zn}$ & $\mathrm{Cd}$ & $\mathrm{\Lambda}$ \\
\hline Témoin & $15,8 \mathrm{a}$ & $23,2 \mathrm{bc}$ & $16,8 \mathrm{a}$ & $29,2 \mathrm{ab}$ \\
$500 \mathrm{~N}$ & $15,9 \mathrm{a}$ & $24,2 \mathrm{bc}$ & $17,3 \mathrm{ab}$ & $30,6 \mathrm{ab}$ \\
$4000 \mathrm{CaO}$ & $17,0 \mathrm{a}$ & $27,5 \mathrm{c}$ & $20,5 \mathrm{c}$ & $30,2 \mathrm{ab}$ \\
$4000 \mathrm{CaO}+$ tourbe & $15,9 \mathrm{a}$ & $26,7 \mathrm{c}$ & $19,2 \mathrm{abc}$ & $29,0 \mathrm{a}$ \\
Tourbe & $15,0 \mathrm{a}$ & $27,0 \mathrm{c}$ & $19,7 \mathrm{bc}$ & $31,5 \mathrm{~b}$ \\
Fe Hydroxyde & $19,5 \mathrm{~b}$ & $19,8 \mathrm{ab}$ & $18,8 \mathrm{abc}$ & $32,1 \mathrm{~b}$ \\
Fe grenail. acier & $64,5 \mathrm{c}$ & $17,5 \mathrm{a}$ & $17,5 \mathrm{ab}$ & $28,8 \mathrm{a}$ \\
& & & & \\
\hline \hline
\end{tabular}

Dans une même colonne, les valeurs qui ne sont pas suivies par une même lettre sont significativement différentes au seuil de $5 \%$.

Within a column, means followed by the same letter are not significantly different at the 0.05 level.

\section{DISCUSSION}

L'absence de pouvoir tampon notable du sol sableux utilisé dans la présence expérimentation explique les variations relativement importantes du pH observées à la suite de lincorporation de sulfate d'ammonium (variation de 0,6 unités de $\mathrm{pH}$ ) ou de carbonate de calcium (variation de 1.7 à 1.9 unités de $\mathrm{pH}$ ). Il n'en reste pas moins que l'éventualité de telles variations ne peut être écartée dans la pratique agricole courante compte tenu de l'ordre de grandeur des quantités de matières fertilisantes qui ont été mises en œuvre pour les provoquer et de la représentativité du type de sol expérimenté (sol soumis à la monoculture intensive du maïs dans le Sud-Ouest atlantique).

Ces modifications de $\mathrm{pH}$ du sol (acidification par l'engrais azoté ammoniacal ou alcalinisation par l'apport de carbonate de calcium) ont provoqué des variations hautement significatives de la biodisponibilité effective des métaux lourds préexistant dans le sol exprimée en terme de concentration dans le végétal (tabl. 9). Ces variations sont bien traduites par l'efficacité de $\mathrm{BaCl}_{2} 0,1 \mathrm{~N}$ ou $\mathrm{CaCl}_{2} 0,1 \mathrm{~N}$ fonctionnant comme réactifs d'échange contrairement à $\mathrm{HCl} 0,1 \mathrm{~N}$ qui, en raison de sa trop grande agressivité, ne permet pas une discrimination valable des différents traitements (tabl. 9).

TABLEAU 9

Coefficient de corrélation entre la teneur en métal du ray-grass, le $\mathrm{pH} d u$ sol et la quantité de métal extraite par $\mathrm{BaCl}_{2} 0,1 \mathrm{~N}, \mathrm{CaCl}_{2}, 0,1 \mathrm{~N}$ et $\mathrm{HCl}(0, I \mathrm{~N}$.

Correlation among plant metal content, soil $\mathrm{pH}$ and the $0.1 \mathrm{~N} \mathrm{BaCl}_{2}$ $0.1 \mathrm{~N} \mathrm{CaCl}$ or $0.1 \mathrm{~N} \mathrm{HCl}$ extractable metal.

\begin{tabular}{ccccc}
\hline \hline Métal & $\mathrm{pH} \mathrm{du} \mathrm{sol}$ & $\mathrm{BaCl}_{2} 0,1 \mathrm{~N}$ & $\mathrm{CaCl}_{2} 0,1 \mathrm{~N}$ & $\mathrm{HCl} 0,1 \mathrm{~N}$ \\
\hline $\mathrm{Mn}$ & $-0,851^{*}$ & $0,744^{*}$ & 0,609 & $-0,122$ \\
$\mathrm{Zn}$ & $-0,777^{*}$ & $0,842^{*}$ & $0,854^{*}$ & $-0,306$ \\
$\mathrm{Cd}$ & $-0,866^{*}$ & $0,826^{*}$ & $0,800^{*}$ & $-0,411$ \\
$\mathrm{Ni}$ & $-0,929^{*}$ & $0,919^{*}$ & $0,923^{*}$ & $-0,472$ \\
& & & & \\
\hline
\end{tabular}

$*$ = significatif à $5 \%$.

* Significant at the 0.05 level.

Il n'en reste pas moins que si $\mathrm{BaCl}_{2} 0,1 \mathrm{~N}$ ou $\mathrm{CaCl}_{2} 0,1 \mathrm{~N}$ autorisent une première approche de la biodisponibilité des métaux du sol, la limite de leur pouvoir prédictif apparait clairement quand on constate, par exemple, que l'abaissement de l'absorption du cadmium à la $6^{\mathfrak{C}}$ coupe de ray-grass (tabl. 5), en présence de tourbe ou d'hydroxyde de fer, ne se traduit pas par une diminution concomitante du caractère échangeable de ce métal (tabl. 6 et 7). A l'inverse, la diminution très sensible de solubilité du zinc dans les 2 solutions salines quand on enrichit le sol avec de l'hydroxyde de fer n'a aucune incidence sur l'absorption du métal par la culture-test. Ceci confirme bien que des facteurs autres que la seule mobilité, exprimée en termes de paramètres physico-chimiques, doivent être pris en compte pour une meilleure compréhension de la biodisponibilité observée. Ainsi, si l'on se réfère aux résultats fournis par le présent travail, il est probable que le fer joue un rôle antagoniste non négligeable dans les processus purement physiologiques régissant l'absorption du cadmium, rôle qui ne peut être mis en évidence par un réactif d'extraction.

Par ailleurs, la présente étude montre que l'effet des variations de biodisponibilité des métaux lourds sur la production végétale peut être masqué par des phénomènes ayant la même origine que celle provoquant précisément la variation de biodisponibilité ; en effet, si 
l'apport d'azote ammoniacal a fortement accru, par acidification, la biodisponibilité de métaux potentiellement phytotoxiques, la phytotoxicité a pu être masquée par l'effet bénéfique du fertilisant, lors des premières coupes notamment. A l'inverse, l'incorporation de carbonate de calcium a considérablement diminué la biodisponibilité des métaux mais elle a aussi abaissé du même coup celle du manganèse, dont la concentration dans le végétal est passée au-dessous du seuil de carence, avec diminution corrélative du rendement.

\section{TABLEAU 10}

Coefficient de corrélation entre production de matière sèche de ray-grass enregistrée pour les differents traitements et teneur en métaux do la plante.

Correlation between dry matter yield of ryegrass and plant metal content.

\begin{tabular}{llll}
\hline \hline & Coupe 1 & Coupe 2 & Coupe 6 \\
\hline Manganèse & $0,764^{* * *}$ & $0,439^{* *}$ & $-0,318$ \\
Zinc & $0,653^{* * *}$ & 0,178 & $-0,355$ \\
Cadmium & $0,577^{* * *}$ & 0,262 & $-0,638^{* * *}$ \\
Nickel & $0,681^{* * *}$ & $0,517^{* *}$ & $-0,522 * *$ \\
& & & \\
\hline \hline
\end{tabular}

Significatif : * à $5 \% ; * *$ à $1 \% ; * * *$ à 1 p. 1000

Significant at: ${ }^{*}=<0.05 ;{ }^{*}=<0.01 ; * * *=0.001$

Malgré ces difficultés d'interprétation (attribuables à la complexité des mécanismes intervenant dans le transfert des métaux du sol au végétal), il semble assez clair que la plus grande production de matière sèche de ray-grass, observée lors de la dernière coupe notamment, dans les vases de végétation contenant le sol additionné de tourbe, d'hydroxyde de fer ou de grenailles d'acier, soit à mettre en relation avec une baisse de la biodisponibilité (tabl. 10) des métaux phytotoxiques ( $\mathrm{Cd}$ et $\mathrm{Ni}$ ) induite par la présence de ces amendements.

\section{CONCLUSION}

Le $\mathrm{pH}$ et, par conséquent, les facteurs qui peuvent agir sur cette caractéristique du sol jouent de toute évidence un rôle déterminant dans la biodisponibilité des métaux lourds incorporés au sol par l'utilisation de certains déchets organiques comme les boues de station d'épuration. Cependant, la possibilité d'une certaine maîtrise du comportement des métaux dans le sol par l'utilisation d'autres artifices que ceux agissant sur le $\mathrm{pH}$ ne peut être ignorée. Dans cet ordre d'idées, la mise en relief, au cours de ce travail, de l'effet antagoniste exercé par des composés du fer vis-à-vis de la biodisponibilité de certains métaux lourds tels que le cadmium pourrait avoir des retombées pratiques intéressantes en matière de lutte contre la pollution métallique des sols.

Reçu le 21 jamiler 1988. Accepté le 14 atôt 1988.

\section{REMERCIEMENTS}

Les auteurs expriment leurs plus vifs remerciements à M. JeanPierre Soyer (Station d'Agronomie de Bordeaux) pour ses conseils éclairés et sa participation active au traitement statistique des résultats.

Cette recherche a bénéficié dune aide financière du Ministère de l'Environnement (S.R.E.T.I.E.), Convention d'étude $n^{\circ} 85.271$.

\section{RÉFÉRENCES BIBLIOGRAPHIQUES}

\begin{abstract}
Abruna F., Chandler J. V., Pearson R. N., 1964. Effects of liming on yiclds and composition of heavily fertilized grasses and on soil properties under humid tropical conditions. Soil Sci. Proc., 28, 657-661
\end{abstract}

Adams F., Pearson R. W., 1969. Neutralizing soil acidity under Bermuda-grass sod Soil Sci. Proc., 33, 737-742.

Adams W. E., Pearson R. W., Jackson W. A., MacCreery R. A., 1967. Influence of limestone and nitrogen on soil pH and coastal Bermudagrass yield. Agron. J., 59, 450-453.

Ames J. W., 1921. Solvent action of nitrification and sulfofication. Bull. Ohio Agric. Exper. Station, 351, 223-257.

Ayres A. S., Hagihara H. H., Stanford G., 1965. Significance of extractable aluminium in Hawaian sugar-cane soils. Soil Sci. Proc., 29, 387-392.

Fiskell J. G. A., 1965. The nature of soil acidity released by several fertilizer materials and fertilizers near the zone of placement. Soil Crop Sci. Soc. Fla. Proc., 25, 173-190.

Fu-Hsian Chang, Broadbent F. E., 1980. Effect of nitrification on movement of trace metals in soil columns. J. Environ. Qual., 9, 4, 587-592.

Hoyt P. B., Henning M. F., 1982. Soil acidification by fertilizers and longcvity of lime applications in the Peace River region. Can. J. Soil Sci., 62, 1, 155-163.

Intrawech A., Stone L. R., Ellis R. Jr., Whitney D. A., 1982. Influence of fertilizer nitrogen source on soil physical and chemical properties. Soil Sci. Soc. Amer. J., 46, 4, 832-836.
Juste C., Soldâ P., 1985. Effect of a long term sludge disposal on cadmium and nickel toxicity to a continuous maize crop. Proc. $4 t h$ Intern. Simp. "Processing and use of organic sludge and liquid agricultural wastes ", Roma, 336-347. Riedel Publish. C. Dordrecht.

Juste C., Tauzin J., 1986. Evolution du contenu en métaux lourds d'un sol de limon maintenu en jachère nue après 56 années d'application continue de divers engrais et amendements. C. R. Acad. Agric. Fr., 72, n'9, 739-746

Linères M., Juste C., 1980. Mise au point de tests agronomiques légers permettant de déterminer simultanément la phytotoxicité globale de boues de station d'épuration et la disponibilité pour les plantes des métaux qu'elles contiennent. Convention Ministire Environnement/I.N.R.A. $\mathrm{n}^{\circ} 77-145,26 \mathrm{p}$. + annexes.

Meguelatti N., Robbe D., Marchandise P., Astruc M., 1983. Intérêt des minćralisations sélectives pour le suivi des pollutions métalliques associćes aux sédiments. J. Fr. $H_{y}$ d drol., 7-10.

Sauerbeck D. R., Styperek P., 1985. Long-term effects of contaminants. Proc. 4th Intern. Simp. "Processing and wse of organic sludge and liquid agricultural wastes", Rome, 318-325. Rieds/ Publish. C ${ }^{\circ}$. Dordrecht.

Siman A., Cradock F. W., Nicholls P. J., Kirton H. C., 1971. Effect of calcium carbonate and ammonium sulfate on manganese toxicity in an acid soil. Austr. J. Agric. Res., 22, 201-214.

Sluijmans C. M. J., 1970. Influence of fertilizer upon the liming status of the soil. Zeitschr. Pflan-. Bodenk., 126, 97-103. 